\title{
Erratum to: Distal pain and carpal tunnel syndrome diagnosis among cashiers: a longitudinal study
}

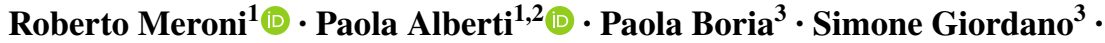 \\ Guido Cavaletti ${ }^{1}$ (B)
}

Published online: 27 July 2017

(C) Springer-Verlag GmbH Germany 2017

\section{Erratum to: Int Arch Occup Environ Health DOI 10.1007/s00420-017-1237-8}

All the tables cited in the article were not included in the original publication. Tables 1, 2, 3, 4 and 5 are included in this erratum.

The original article was corrected.

The online version of the original article can be found under doi:10.1007/s00420-017-1237-8.

\section{Guido Cavaletti}

guido.cavaletti@unimib.it

1 Dipartimento di Medicina e Chirurgia, University of MilanoBicocca, School of Medicine and Surgery, Via Cadore 48, 20900 Monza, Italy

2 Ph.D. Program in Neuroscience, University of Milano-Bicocca, School of Medicine and Surgery, Via Cadore 48, 20900 Monza, Italy

3 S.G.B.P. Soluzioni Gestionali per il Benessere Psicofisico, Via Giuseppe Frua 21/8, 20146 Milan, Italy 
Table 1 Demographic data and exposure details of the whole population
Table 2 Employment years in the supermarket for the subjects with and without positive CTS

\begin{tabular}{|c|c|c|c|c|c|c|}
\hline & $\mathrm{Age}^{\mathrm{a}}$ & $p^{\mathrm{b}}$ & BMI & $p^{\mathrm{b}}$ & $\begin{array}{l}\text { Hours at the cashier } \\
\text { per year }^{\text {a }}\end{array}$ & $p^{\mathrm{b}}$ \\
\hline \multicolumn{7}{|c|}{ Carpal tunnel syndrome } \\
\hline Yes (17) & $42(8)[41]$ & 0.305 & $24(4)[23]$ & 0.311 & 819 (234) [776] & 0.289 \\
\hline No (132) & 41 (7) [40] & & 23 (4) [22] & & 877 (352) [954] & \\
\hline
\end{tabular}

Nerve conduction studies were used to stratify population according to carpal tunnel presence, any grade (see text for reference: Padua et al. 1997)

$B M I$ body mass index

${ }^{a}$ Mean (SD), and [median] values

${ }^{\mathrm{b}}$ Results of the Independent-Sample Mann-Whitney $U$ test

\begin{tabular}{|c|c|c|c|c|c|c|c|}
\hline & \multicolumn{7}{|c|}{ Employment years in the supermarket } \\
\hline & $1-3$ years & $4-6$ years & $7-9$ years & $10-15$ years & $16-20$ years & $>20$ years & Total \\
\hline \multicolumn{8}{|c|}{ Carpal tunnel syndrome } \\
\hline \multicolumn{8}{|c|}{ Yes } \\
\hline \# & 0 & 1 & 2 & 2 & 9 & 3 & 17 \\
\hline$\%$ & 0 & 5.9 & 11.8 & 11.8 & 52.9 & 17.6 & 100.0 \\
\hline \multicolumn{8}{|l|}{ No } \\
\hline \# & 3 & 7 & 17 & 20 & 71 & 14 & 132 \\
\hline$\%$ & 2.3 & 5.3 & 12.9 & 15.2 & 53.8 & 10.6 & 100.0 \\
\hline \multicolumn{8}{|l|}{ Total } \\
\hline$\#$ & 3 & 8 & 19 & 22 & 80 & 17 & 149 \\
\hline$\%$ & 2.0 & 5.4 & 12.8 & 14.8 & 53.7 & 11.4 & 100.0 \\
\hline
\end{tabular}

Employment years have been stratified into six classes. Nerve conduction studies were used to stratify population according to carpal tunnel presence, any grade (see text for reference: Padua et al. 1997)

Fisher's exact test $=1.362, p=0.935$ 


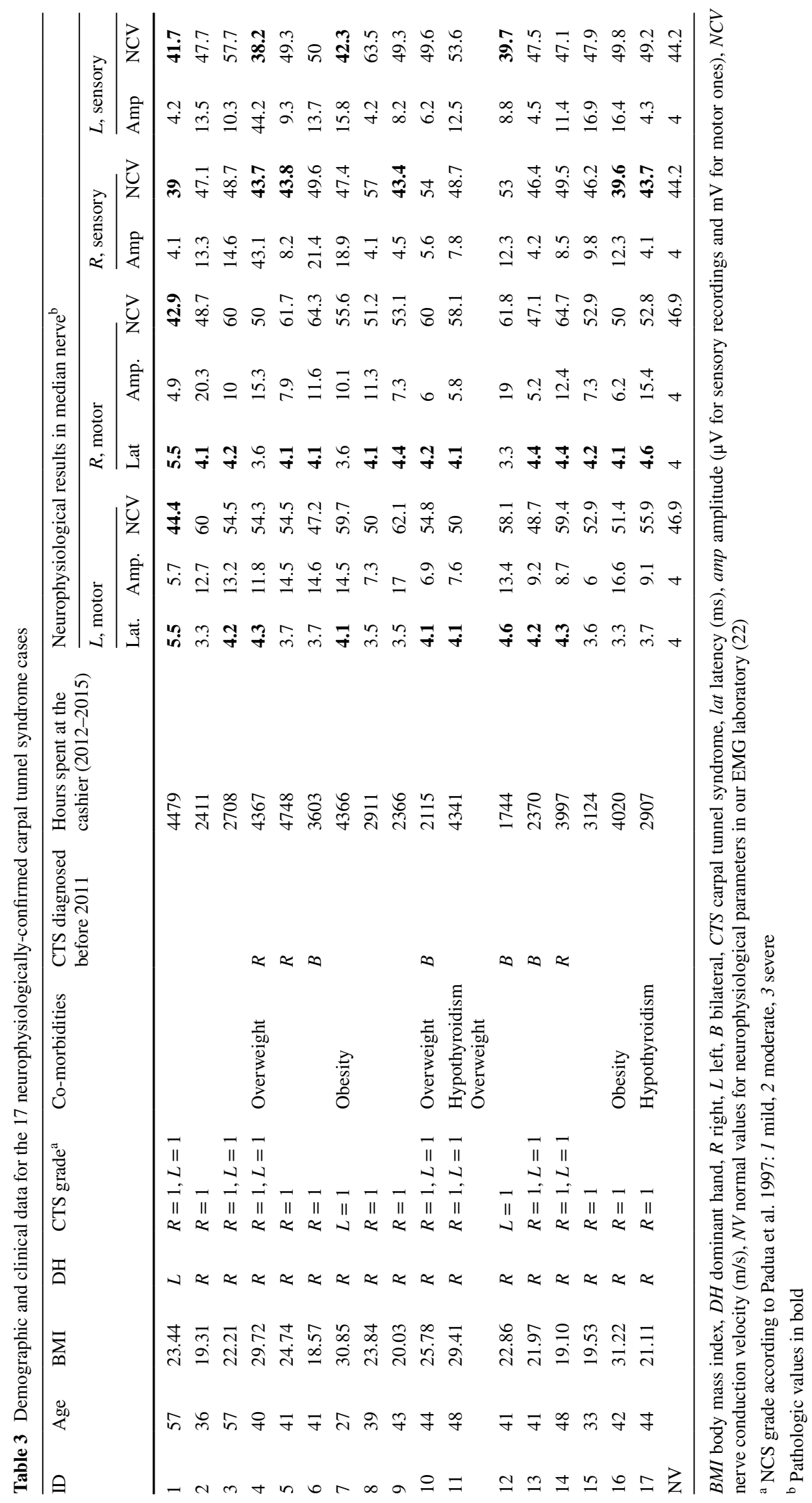


Table 4 Data regarding previous exposure to work at risk for CTS are reported

\begin{tabular}{clll}
\hline & \multicolumn{2}{c}{ Positive for CTS } & Total \\
\cline { 2 - 3 } & Yes & No & \\
\hline $\begin{array}{c}\text { Previous work at risk for CTS } \\
\text { Yes }\end{array}$ & & \\
$\#$ & 7 & 63 & 70 \\
$\%$ & 10.0 & 90.0 & 100.0 \\
No & & & \\
$\#$ & 10 & 69 & 79 \\
$\%$ & 12.7 & 87.3 & 100.0 \\
Total & & & \\
$\#$ & 17 & 132 & 149 \\
$\%$ & 11.4 & 88.6 & 100.0 \\
\hline
\end{tabular}

Nerve conduction studies were used to stratify population according to carpal tunnel presence, any grade (see text for reference: Padua et al. 1997)

Odds ratio $=0.767,95 \% \mathrm{CI}=0.275-2.136$
Table 5 Comorbidity frequency in the whole study population

\begin{tabular}{clll}
\hline & \multicolumn{2}{c}{ Positive for CTS } & Total \\
\cline { 2 - 3 } & Yes & No & \\
\hline $\begin{array}{c}\text { Presence of comorbidity } \\
\text { Yes }\end{array}$ & & \\
$\#$ & 6 & 12 & 18 \\
$\%$ & 33.3 & 66.7 & 100.0 \\
No & & & \\
$\#$ & 11 & 120 & 131 \\
$\%$ & 8.4 & 91.6 & 100.0 \\
Total & 17 & & \\
$\%$ & 11.4 & 132 & 149 \\
\hline
\end{tabular}

Nerve conduction studies were used to stratify population according to carpal tunnel presence, any grade (see text for reference: Padua et al. 1997)

Odds ratio $=5.455,95 \% \mathrm{CI}=1.713-17.360$ 